This document is the Accepted Manuscript version of a Published Work that appeared in final form in Crystal Growth and Design, copyright (c) American Chemical Society after peer review and technical editing by the publisher. To access the final edited and published work see:

https://dx.doi.org/10.1021/acs.cgd.6b01115. 


\title{
A first Cyclodextrin-Transition Metal Coordination Polymer
}

\author{
Heng Xu, ${ }^{\dagger}$ Sabina Rodríguez-Hermida, ${ }^{\dagger}$ Javier Pérez-Carvajal, ${ }^{\dagger}$ Jordi Juanhuix, $^{\dagger}$ Inhar Imaz, ${ }^{\dagger,}$ \\ Daniel Maspoch, $\$, \dagger^{*}$ \\ ${ }^{\dagger} \mathrm{ICN}_{2}$ - Institut Català de Nanociencia i Nanotecnologia, CSIC and The Barcelona Institute of \\ Science and Technology, Campus UAB, 08193 Bellaterra Barcelona, Spain, ${ }^{\ddagger}$ ALBA Synchrotron, \\ o829o Cerdanyola del Vallès, Barcelona, Spain, ${ }^{\S}$ ICREA, Pg Lluís Companys 23, o8010 Barcelona, \\ Spain
}

\section{Supporting Information Placeholder}

\begin{abstract}
The synthesis, X-ray crystallographic structure and water uptake measurements of the first coordination polymer made of a cyclodextrin and a transition metal ion (in this case, $\mathrm{Cu}^{2+}$ ) is shown. This coordination polymer is made by connecting paddle-wheel $\mathrm{Cu}^{2+}$ units through a $\gamma^{-}$ cyclodextrin functionalized with eight carboxylate groups, is stable in water, and shows selective water-induced reversible structural transformations.
\end{abstract}

Coordination polymers $(\mathrm{CPs})^{1}$ can be defined as hybrid inorganic-organic networks extended in one, two or three dimensions through coordination bonds. Since the introduction of the design concept by Robson in 1990's, the number of synthesized CPs has experienced an exponential growth. ${ }^{2}$ Especially with the advent of the rich variety of permanent porosity properties found in CPs (known as porous CPs (PCPs) or Metal-Organic Frameworks (MOFs), CPs show today promise for myriad applications including gas adsorption, catalysis, separation, sensing and drug delivery, among many others. ${ }^{3-7}$ In this area, the continuous incorporation of new organic ligands has allowed developing novel CPs and fine-tune their properties. Beyond the classical rigid multitopic carboxylate and N-donor based molecules that constitutes the vast majority of ligands studied so far, it recently exists an increasing interest in introducing more complex functional ligands to form CPs because they can directly incorporate their own functionalities in the coordination structures. For example, nanometer-sized molecules such as electro-active fullerenes, ${ }^{8}$ catalytic clusters such as polyoxometalates ${ }^{9}$ and classical inclusion molecules such as calixarenes ${ }^{10}$ or cyclodextrines ${ }^{11}$ (CDs) have started to be used as functional ligands for the formation of new CPs.

CDs are formed by six $(\alpha-C D)$, seven $(\beta-C D)$ or eight $(\gamma-$ CD) glucopyranose units bound via $-1,4^{-}$glycosidic linkages. They show a toroid structure that is internally hydrophobic and externally hydrophilic. These characteristics make them very useful for encapsulating functional molecules in their cavities. For example, CDs able to encapsulate drugs can today be found in almost forty different pharmaceutical
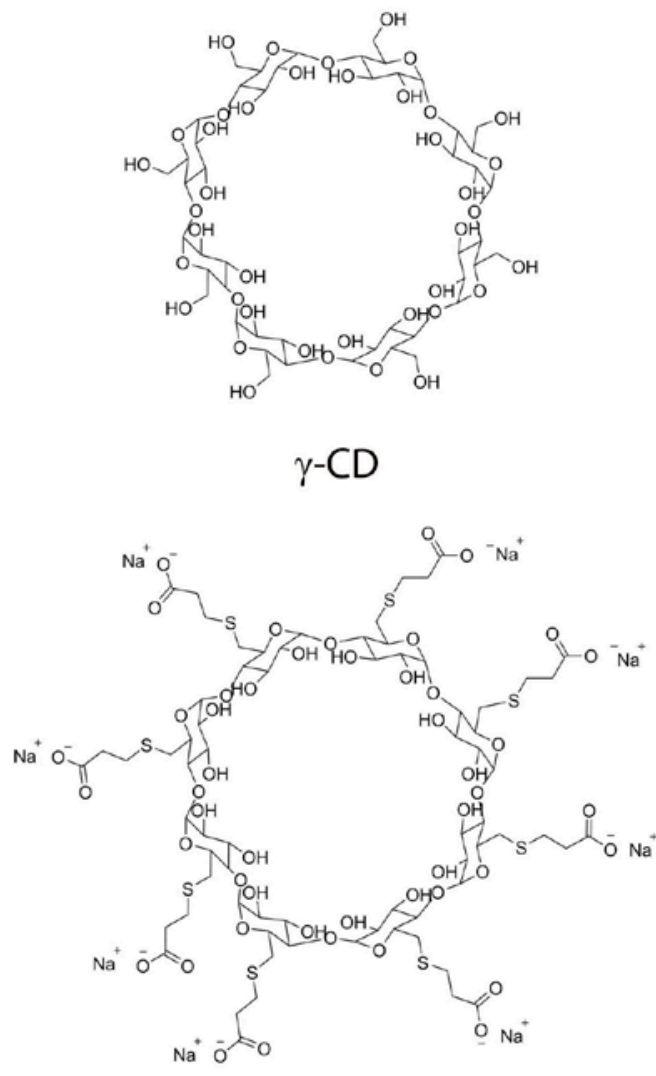

SD

Scheme 1. Illustration of $\gamma-C D$ and SD.

products. ${ }^{12}$ However, even though CDs have been studied for more than hundred years, their coordination chemistry with metal ions has not been widely explored due to the difficulty to crystallize well-defined systems and get correct structural data. In this area, some examples in which transition metal ions (e.g. $\mathrm{Cu}(\mathrm{II}), \mathrm{Zn}(\mathrm{II}), \mathrm{Co}(\mathrm{II}), \mathrm{Mn}(\mathrm{II})$, etc.) connect $\mathrm{CDs}$ 
forming sandwich or bucket-wheel type structures have been reported. ${ }^{13}$ More recently, Stoddart and coworkers has shown the possibility to assemble CDs with alkali metal ions to form $\mathrm{PCP} / \mathrm{MOF}$. In 2010, they reported a first $3 \mathrm{D}$ porous $\mathrm{MOF}$ (called CD-MOF-1) built up from combining $\gamma$-CD and $\mathrm{K}^{+}$ ions. ${ }^{10}$ The X-ray crystal structure of CD-MOF-1 revealed the assembly of $(\gamma-C D)_{6}$ through $\mathrm{K}-\mathrm{OH}$ linkages forming a bodycentered cubic structure, wherein each $\mathrm{K}^{+}$ions connects two CDs that occupy the faces of the cube. Later, similar CDMOFs were obtained using salts of $\mathrm{Rb}^{+}(\mathrm{CD}-\mathrm{MOF}-2)$ and $\mathrm{Cs}^{+}$ (CD-MOF-3). Interestingly, both CD-MOF1 and CD-MOF-2 showed BET surface areas of $1220 \mathrm{~m}^{2} \cdot \mathrm{g}^{-1}$ and $1110 \mathrm{~m}^{2} \cdot \mathrm{g}^{-1}$, respectively.

The inclusion properties of CDs offer interesting properties to CD-based MOFs. For instance, Stoddart et al. reported the highly selective adsorption of $\mathrm{CO}_{2}$ by CD-MOF-2, by what is believed to be reversible carbon fixation inside the $\gamma$-CD involving carbonate formation and decomposition at room temperature. In addition, CD-MOF-1 and -2 demonstrated their ability to separate a wide variety of mixtures, including ethylbenzene from styrene, haloaromatics, terpinenes, pinenes, and other chiral compounds. ${ }^{14-16}$

Although these many interesting properties, one of the limitations of these CD-based MOFs is still the weakness of the bond that supports these frameworks; that is, the bond between the hydroxyl groups of the $\gamma-C D$ and the alkali metal ions. This provokes that these MOFs can be instantly dissolved in an aqueous environment or degraded when exposed to humidity, greatly hampering some of their practical applications. To stabilize these CD-based MOFs, Furukawa et al. have explored the use of ethylene glycol diglycidyl ether to crosslink the CD-MOF-1 producing hydrogels. ${ }^{17}$ More recently, Falcaro et al. proposed a facile method to improve the hydrolytic stability of CD-MOF-1 by incorporating the hydrophobic C6o in their matrices, proving their potential use to encapsulate and release doxorubicin in aqueous media. $^{18}$

Another strategy that should provide robust coordination networks containing $\mathrm{CDs}$ is to strength the coordination bond between the CDs and the metal ions. Here, we report an initial step to achieve this goal by reporting the first example of a CP (hereafter called Cu-SD) made of a $\gamma$-CD and a transition metal ion. For this purpose, we used octakis-[6deoxy-6-(3-mercaptopropanoic sodium)]- $\gamma$-cyclodextrin (SD; also known as org25969 or Sugammadex $\left.{ }^{\circledR}\right)$ as the organic ligand, and $\mathrm{Cu}^{2+}$ as the transition metal ion. SD is an octacarboxylate $\gamma-C D$ in which each one of the eight C6 hydroxy groups of the $\gamma$-CD has been substituted with sulfurylpropionic acid arms (Scheme 1). SD is today a rapid and efficient selective relaxant binding agent approved by FDA, ${ }^{19}$ acting as a trapping agent of the rocuronium bromide; a widely used neuromuscular blocker during anaesthesis. ${ }^{20,21}$

Cu-SD was prepared using a slow diffusion method. An aqueous solution $(1.5 \mathrm{ml})$ containing $\mathrm{SD}(20 \mathrm{mg}$; $0.009 \mathrm{mmol})$ was initially placed into the bottom of a tube. Then, a mixture of $\mathrm{H}_{2} \mathrm{O} / \mathrm{EtOH}(1: 1,4 \mathrm{ml})$ was carefully added on top of this solution as a buffer zone. Finally, an ethanolic solution (1.5 ml) containing $\mathrm{CuCl}_{2} \cdot 2 \mathrm{H}_{2} \mathrm{O}$ (20 mg; $0.117 \mathrm{mmol}$ ) was carefully added on top of the $\mathrm{H}_{2} \mathrm{O} / \mathrm{EtOH}$ mixture. The diffusion proceeded at room temperature for a week in static conditions, resulting in blue cubic crystals suitable for single- crystal X-ray diffraction (SCXRD). The resulting crystals were collected by centrifugation, and washed two times with a $\mathrm{H} 2 \mathrm{O} / \mathrm{EtOH}$ (1:1) mixture ( $2 \mathrm{ml}$ each step) followed by another three times with pure $\mathrm{EtOH}$ ( $2 \mathrm{ml}$ each step). The final yield was $40 \%$ (based on SD).

Crystallographic data was obtained from Cu-SD crystals at the XALOC beamline of the ALBA Synchrotron under synchrotron radiation $(\lambda=0.79474 \AA)$ using a MD2M single axis diffractometer (Maatel, France) and a Pilatus 6M detector (Dectris, Switzerland). ${ }^{22}$ Due to its sensitivity to solvent loss, a crystal of $\mathbf{C u}-\mathbf{S D}$ was measured mounted in a thin glass capillary suspended in the reaction mother liquor (mixture of $\mathrm{H}_{2} \mathrm{O} / \mathrm{EtOH}$ ) and at a temperature of $293 \mathrm{~K}$ (to avoid water congelation). The crystal showed poor diffraction data with reflections of moderate intensity, which were further degraded rapidly after 120 degrees oscillation. This poor density and fast degradation (seen in all measured crystals) could be attributed to the solvent loss. These 120 data frames were indexed, integrated and scaled using the $\mathrm{XDS}^{23}$ and IMOSFLM $^{24}$ programs. Absorption correction was not applied. The structure was solved by direct methods and subsequently refined by correction of $\mathrm{F}_{2}$ against all reflections, using SHELXS2O13 and SHELXL2O13 within the WinGX package. ${ }^{25-27}$ All non-hydrogen atoms were refined with anisotropic thermal parameters by full-matrix least-squares calculations on $\mathrm{F}_{2}$ using the program SHELXL2013. Hydrogen atoms were inserted at calculated positions and constrained with isotropic thermal parameters. The disorder associated with the oxygen atoms of the coordinated water molecules hindered the localization of their hydrogen atoms. One sulphur atom is distorted in two positions with $35 \%$ and $65 \%$ of occupancy. The structure contains some disorder molecules. Attempts to adequately model the disordered water molecules (10 electrons) were unsatisfactory; therefore, the PLATON/SQUEEZE routine was applied to mask out the disordered electron density. ${ }^{28}$ The residual electron density was assigned to 46 water molecules per $\left[\mathrm{Cu}_{4}(\mathrm{SD})\left(\mathrm{H}_{2} \mathrm{O}\right)_{4}\right]$ (1854 electrons per unit cell). Crystallographic and refinement data, bond distances and angles and $\mathrm{H}$-bond interactions are listed in Tables $\mathrm{S}_{1}, \mathrm{~S}_{2}$ and $\mathrm{S}_{3}$.

The crystal data reveals the formation of a $\mathrm{CP}$ with formula $\left[\mathrm{Cu}_{4}(\mathrm{SD})\left(\mathrm{H}_{2} \mathrm{O}\right)_{4}\right] \cdot 46 \mathrm{H}_{2} \mathrm{O}(\mathbf{C u}-\mathrm{SD})$ that crystallizes in the tetragonal $\mathrm{P}_{4} \mathrm{n} 2 \mathrm{n}$ space group. $\mathrm{Cu}-\mathrm{SD}$ shows a $2 \mathrm{D}$ structure built up from linking the $\mathrm{SD}$ units through dinuclear $\mathrm{Cu}_{2}(\mathrm{OOC})_{4}\left(\mathrm{H}_{2} \mathrm{O}\right)_{2}$ paddle wheel clusters. Note here that other coordination systems found in the literature with transition metal ions are discrete structures, which are further extended via alkali metals or supramolecular bonds. ${ }^{9}$ In $\mathbf{C u}-$ $\mathrm{SD}$, all $\mathrm{Cu}^{2+}$ ions adopt a square base pyramid geometry (Addison parameter $\tau$ values within the 0.045-0.056 range) coordinated to four oxygen atoms belonging to four carboxylic groups and to one water molecule occupying the apical position (Figure 1a). The equatorial $\mathrm{Cu}-\mathrm{O}$ distances vary between 1.92(1) $\AA$ and 1.98(1). These distances are much shorter than the $\mathrm{K}-\mathrm{OH}$ distances (between 2.79 and 2.84) found in CD-MOF-1, ${ }^{6}$ but are comparable to the $\mathrm{Cu}-\mathrm{O}$ distances observed in the above-mentioned sandwich type $\mathrm{Cu}$ $\mathrm{CD}$ systems (e.g. $\mathrm{Li}_{3}\left[\mathrm{Li}_{3} \mathrm{Cu}_{3}\left(\alpha-\mathrm{CDH}_{-6}\right)_{2}\right]$ and $\mathrm{Na}_{3}\left[\mathrm{Na}_{3} \mathrm{Cu}_{3}(\alpha-\right.$ $\left.\mathrm{CDH}_{-6}\right)_{2}$ ] sandwiches, showing $\mathrm{Cu}-\mathrm{O}$ distances between 1.90 A and $1.98 \AA)^{29}$ 
a)

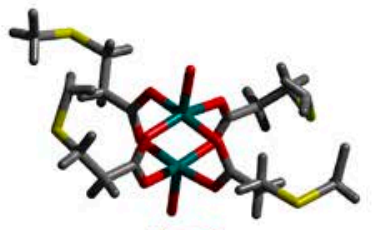

b)

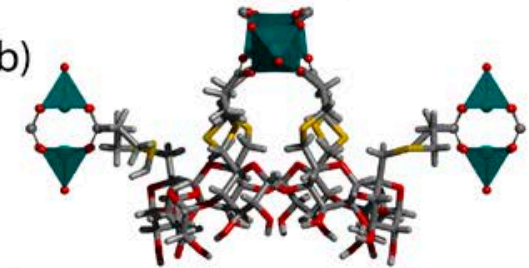

e)

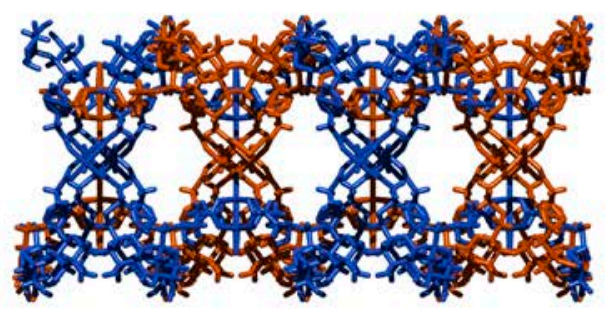

c)

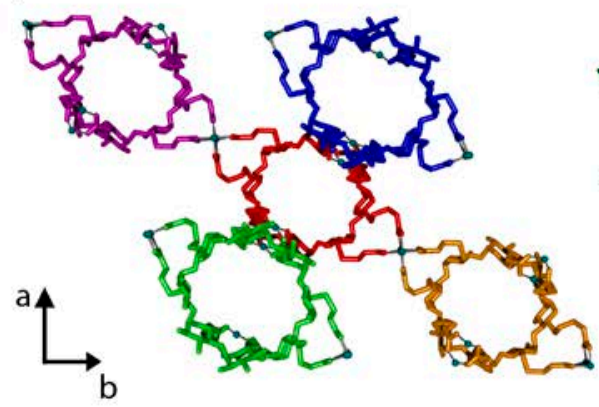

d)

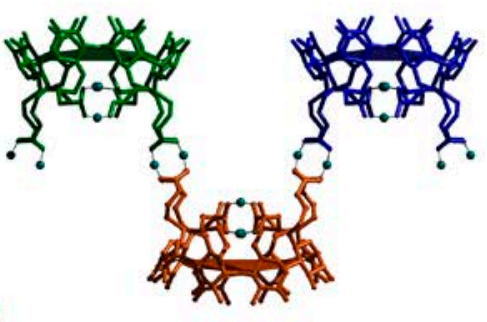

f)

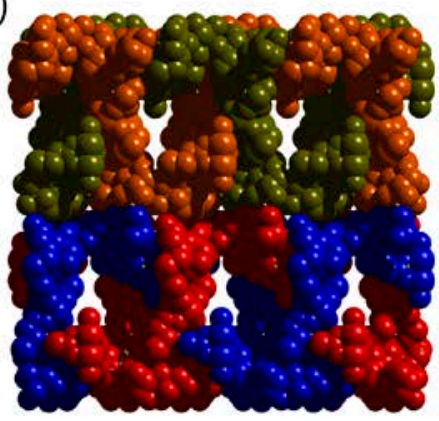

g)

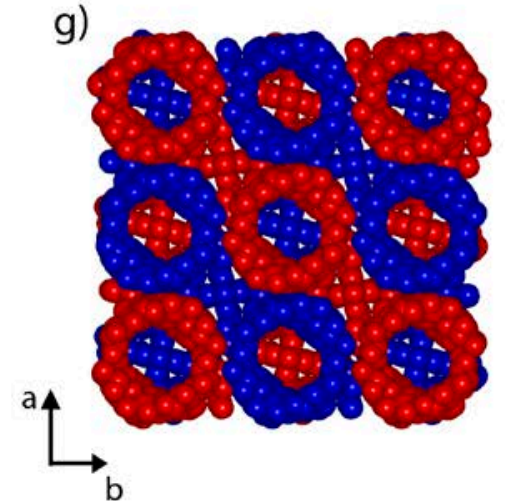

Figure 1. a) Illustration of the paddle-wheel $\mathrm{Cu}^{2+}$ unit. b) Illustration showing that each SD links to four paddle-wheel $\mathrm{Cu}^{2+}$ units. The darkcyan tetrahedral represents the coordination environment of $\mathrm{Cu}^{2+}$ ions. c) View showing that each SD (red) is connected to four other SD linkers (pink, blue, orange and green) through four paddle-wheel $\mathrm{Cu}^{2+}$ units along the $<1 \overline{1} \mathrm{o}>$ direction. d) Representation showing the coordination of each SD along the $<1 \overline{1} 0>$ direction. e) Representation of the 2 -fold interpenetrated layer. f,g) Space-filling view of the packing of the 2-fold interpenetrated layer, showing an offset ABA'B' packing. Each individual layer is highlighted with a different color. In a) and b), color code: O: red; S: yellow; C: grey; and Cu: darkcyan.

In the extended structure, each SD ligand is coordinated to eight $\mathrm{Cu}^{2+}$ ions. The eight flexible 3-mecaptopropionate arms are involved in the formation of four paddle wheel units involving four other SD ligands (Figure $\mathrm{ib}$ and $\mathrm{c}$ ). Each SD ligand shows four carboxylate arms pointing along the equatorial plane of the SD core, whereas the four remaining ones are pointing up or down of this equatorial plane with an angle of 70,5 o (Figure $1 \mathrm{~d}$ ). This connectivity results in the formation of 2 -fold interpenetrated layers running along the $a b$ plane (Figure 1e). The interpenetrated layers establish $\mathrm{H}$ bond interactions between them, involving the coordinated water molecule $\left(\mathrm{O}_{3} \mathrm{w}\right)$ and an $\mathrm{O}$ atom belonging to a carbox- ylate group (Figure $\mathrm{S}_{1}$ and Table $\mathrm{S}_{3}$ ). The association between these interpenetrated layers yields to an offset ABA'B' packing (Figure if and g). The multiple hydroxyl groups of the SD ligand are pointed outer the layers, allowing to establish intermolecular $\mathrm{H}$-bond interactions between the sheets along the $c$ axis (Table $\mathrm{S}_{3}$ ). Cu-SD displays a solvent accessible volume of $41.1 \%$ (corresponding to $5837.3 \AA^{3}$ ) calculated using PLATON. This free volume is mainly located inside the two interpenetrated sheets (Figure S2), and it is filled of highly disordered water molecules (SQUEEZE results show a total of 46 guest water molecules). 
a)

b)
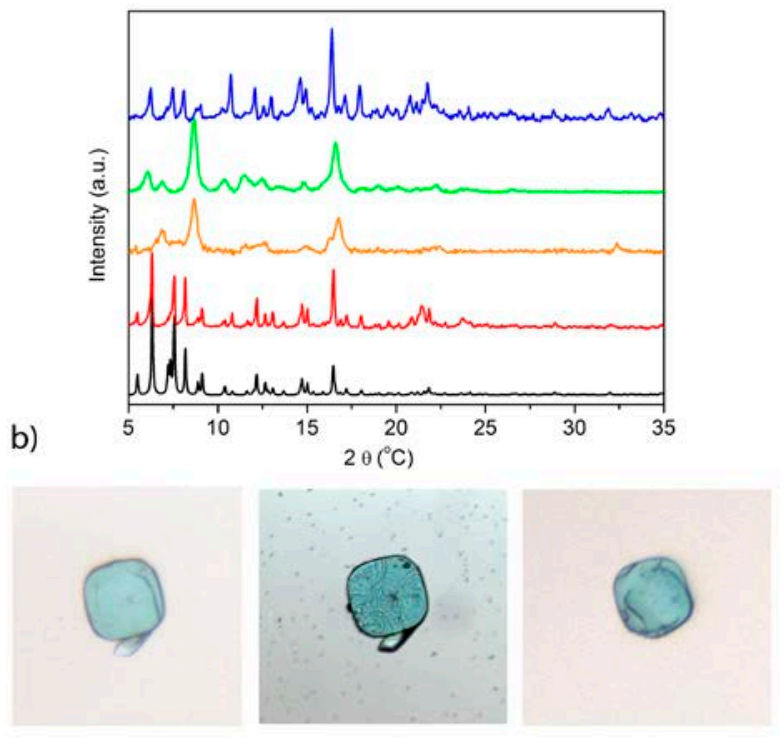

c)

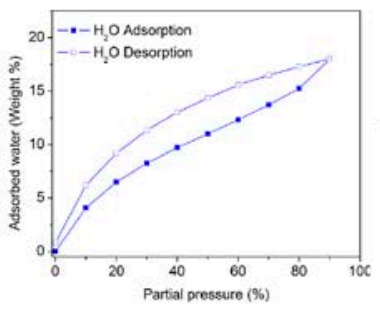

d)

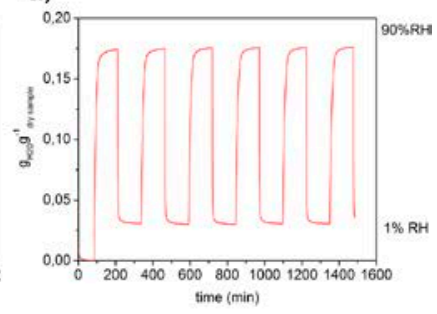

Figure 2. a) XRPD patterns of as synthesized Cu-SD (red), dried $\mathbf{C u}-\mathrm{SD}$ (orange), $\mathrm{Cu}-\mathrm{SD}$ heated under vacuum at $8 \mathrm{o}^{-}$ $C$ (green), and $\mathbf{C u}-\mathbf{S D}$ reinmersed in water (blue), compared to simulated XRPD powder pattern (black). b) Photographs of a single crystal of as-synthesized $\mathbf{C u}$-SD (left), dried CuSD showing cracking (center), and dried Cu-SD reinmersed in water (right). c) Water adsorption and desorption isotherm of Cu-SD. (d) Six consecutive cycles of water adsorption/desorption showing the perfect reversibility of the sorption process.

As expected from what we observed during the crystal structure determination by SCXRD, crystals of $\mathbf{C u}-\mathbf{S D}$ rapidly lost guest molecules and underwent structural transformations when they were dried at room temperature. Powder $\mathrm{X}$-Ray diffraction (PXRD) performed on the resulting crystals revealed a shift and a widening of most of the peaks, which was indicative of a loss of crystallinity as well as of structural re-arrangements (Figure 2a). Unfortunately, cracking of the crystals during the drying process prevented us to solve the crystal structure corresponding to the dried phase by SCXRD (Figure 2b). Guest water molecules were fully removed from air-dried Cu-SD by heating the dried crystals under vacuum ( 0.05 torr) at $80^{\circ} \mathrm{C}$ for $\mathrm{i} \mathrm{h}$, as confirmed by elemental analysis and TGA measurements (Figure $\mathrm{S}_{3}$ ). Here, the PXRD pattern showed no significant differences in comparison to the dried sample at room temperature (Figure 2a), confirming that no further changes occur during this treatment. Attempts to study the adsorption of $\mathrm{N}_{2}$ at $77 \mathrm{~K}$ and $\mathrm{CO}_{2}$ at $195 \mathrm{~K}$ showed no evidence for gas uptake (Figure $\mathrm{S}_{4}$ ). However, these crys- tals (either dried at room temperature or heated under vacuum at $80^{\circ} \mathrm{C}$ ) showed evidence for the reversible uptake of water molecules. Indeed, when we reinmersed these crystals in water for one hour, PXRD analysis of the resulting crystals revealed that the initial $\mathbf{C u}-\mathbf{S D}$ phase is recovered, demonstrating that the structural transition is reversible (Figure $2 \mathrm{a}$ and b). Remarkably, this reversible structural transition is selective to water since the PXRD of an air-dried Cu-SD sample soaked in liquid ethanol, dimethylformamide and acetonitrile showed that initial crystal structure cannot be recovered (Figure $\mathrm{S}_{5}$ ).

As this reversible transition must involve the loss and gain of many water molecules, we then investigated the water sorption properties of $\mathbf{C u}-\mathbf{S D}$ (Figure $2 \mathrm{C}$ and d). The collected water vapor sorption isotherm of a $\mathbf{C u}-\mathbf{S D}$ sample heated under vacuum at $80^{\circ} \mathrm{C}$ showed an adsorption branch representative of a type II trend. ${ }^{25}$ The maximum water uptake was $0.175 \mathrm{~g}$ water / g Cu-SD at a $\mathrm{RH}$ of $90 \%$, which correspond to 28 water molecules per $\left[\mathrm{Cu}_{4}(\mathrm{SD})\left(\mathrm{H}_{2} \mathrm{O}\right)_{4}\right]$. We then performed six water sorption/desorption cycles by alternately exposing the dry material to humid (90\% RH) and dry $(1 \%$ $\mathrm{RH}$ ) environments (Figure 2d). Remarkably, the maximum uptake at 90\% RH (ca. 17.5\%) was not significantly modified with the number of cycles, confirming the stability of this framework.

In conclusion, we have reported the use of a $\gamma-C D$ functionalized with eight carboxylate groups to synthesize the first cyclodextrin CP made with a transition metal ion. This $\mathrm{CP}$ shows a $2 \mathrm{D}$ coordination structure, is stable in water, and exhibits reversible structural transformations upon water sorption/desorption. With the description of this first CDtransition metal $\mathrm{CP}$, synthesizing PCPs that incorporates the inclusion-recognition properties of CDs should be feasible.

\section{ASSOCIATED CONTENT}

\section{Supporting Information}

The Supporting Information is available free of charge on the ACS Publications website at DOI:XXXX.

Experimental section; crystallographic data; crystal structure images; TGA; and XRPD.

\section{AUTHOR INFORMATION}

\section{Corresponding Author}

\section{inhar.imaz@icnz.cat}

daniel.maspoch@icn2.cat

Notes

The authors declare no competing financial interest.

\section{ACKNOWLEDGMENT}

This work was supported by the MINECO-Spain through the project PN MAT2012-30994. I.I. thanks the MINECO for a RyC contract. H.X. thanks the Generalitat de Catalunya for a FI grant. ICN2 acknowledges the support of the Spanish MINECO through the Severo Ochoa Centers of Excellence Program, under Grant SEV-2013-0295. 


\section{REFERENCES}

(1) Batten, S. R.; Neville, S. M.; Turner, D. R. Coordination Polymers: Design, Analysis and Application; Royal Society of Chemistry: Cambridge, 2009.

(2) Hoskins, B. F.; Robson, R. J. Am. Chem. Soc. 1989, 111, 5962-5964.

(3) Kitagawa, S.; Kitaura, R.; Noro, S. Angew. Chem. Int. Ed. 2004, 43, 2334-2375.

(4) Long, J. R.; Yaghi, O. M. Chem. Soc. Rev. 2009, 38, 12131214.

(5) DeCoste, J. B.; Peterson, G. W. Chem. Rev. 2014, 114, 5695-5727.

(6) Dou, Z.; Yu, J.; Cui, Y.; Yang, Y.; Wang, Z.; Yang, D.; Qian, G. J. Am. Chem. Soc. 2014, 136, 5527-5530.

(7) Dalgarno, S. J.; Cholewa, P. P. CrystEngComm 2014, 16, 3655-3666.

(8) Peng, P.; Li, F.-F.; Neti, V. S. P. K., Metta-Magana, A. J.; Echegoyen, L. Angew. Chem. Int. Ed. 2014, 53, 160-163.

(9) Miras, H. N.; Vilá-Nadal, L.; Cronin, L. Chem. Soc. Rev. 2014, 43, 5679-5699.

(10) Smaldone, R. A.; Forgan, R. S.; Furukawa, H.; Gassensmith, J. J.; Slawin, A. M. Z.; Yaghi, O. M.; Stoddart, J. F. Angew. Chem., Int. Ed. 2010, 49, 8630-8634.

(11) Dodziuk, H. Cyclodextrins and Their Complexes; WileyVCH: Weinheim, Germany, 2006.

(12) Loftssona, T.; Duchêne, D. Int. J. Pharm. 2007, 329, 1-11.

(13) Prochowicz, D.; Kornowicz, A.; Justyniak, I.; Lewiński, J. Coord. Chem. Rev. 2016, 306, 331-345.

(14) Gassensmith, J. J.; Furukawa, H.; Smaldone, R. A.; Forgan, R. S.; Botros, Y. Y.; Yaghi, O. M.; Stoddart, J. F. J. Am. Chem. Soc. 2011, 133, 15312-15315.

(15) Gassensmith, J. J.; Kim, J. Y.; Holcroft, J. M.; Farha, O. K.; Stoddart, J. F.; Hupp, J. T.; Jeong, N. C. J. Am. Chem. Soc. 2014, 136,8277-8282.
(16) Hartlieb, K. J.; Holcroft, J. M.; Moghadam, P. Z.; Vermeulen, N. A.; Algaradah, M. M.; Nassar, M. S.; Botros, Y. Y.; Snurr, R. Q.; Stoddart, J. F. J. Am. Chem. Soc. 2016, 138, 22922301.

(17) Furukawa, Y.; Ishiwata, T.; Sugikawa, K.; Kokado, K.; Sada, K. Angew. Chem. Int. Ed. 2012, 51, 10566-10569.

(18) Li, H.; Hill, M. R.; Huang, R.; Doblin, C.; Lim, S.; Hill, A. J.; Baba-rao, R.; Falcaro, P. Chem. Commun. 2016, 52, 59735976.

(19) Zhang, M.-Q.; Palin, R.; Bennett, D. J. PCT Int. Appl. WO 0140316 Al, 2001; Chem. Abstr. 20o1, 135, 29151.

(20) Adam, J. M.; Bennett, D. J.; Bom, A.; Clark, J. K.; Feilden, H.; Hutchinson, E.; Palin, R.; Prosser, A.; Rees, D. C.; Rosair, G. M.; Stevenson, D.;. Tarver, G. J.; Zhang, M. Q. J. Med. Chem. 2002, 45, 1806-1816.

(21) Bom, A.; Bradley, M.; Cameron, K.; Clark, J. K.; Egmond, J. V.; Feilden, H.; MacLean, E. J.; Muir, A. W.; Palin, R.; Rees, D. C.; Zhang, M. Q. Angew. Chem. Int. Ed. 2002, 41, 266-270.

(22) Juanhuix, J.; Gil-Ortiz, F.; Cuní, G.; Colldelram, C.; Nicolás, J.; Lidón, J.; Boter, E.; Ruget, C.; Ferrer, S.; Benach, J. J. Synchrotron Radiat. 2014, 21, 679-689.

(23) Kabsch, W. XDS-Acta Crystallogr. D Biol. Crystallogr. 2010, 66, 125-132.

(24) Leslie, AG. Acta Crystallogr. D Biol. Crystallogr. 2006, 62, 48-57.

(25) Sheldrick, G. M.; Dauter, Z.; Wilson, K. S.; Hope, H.; Sieker, L. C. Acta Crystallogr. D Biol. Crystallogr. 1993, 49, 1823.

(26) Sheldrick, G. Acta Crystallogr. C Struct. Chem. 2015, 71, 3-8.

(27) Farrugia, L. J. J. Appl. Cryst. 2012, 45, 849-854.

(28) Spek, A. L. PLATON-A Multipurpose Crystallographic Tool, Utrecht University, Utrecht (The Netherlands), 2001.

(29) Klüfers, P.; Piotrowski, H.; Uhlendorf, J. Chem. Eur. J. 1997, 3, 601-6o8. 